\title{
Efficient Data Caching Based On Fuzzy Decision Routing In Vehicle Disruption Tolerant Networks
}

\author{
G. Menaka ${ }^{1}$, Mr.Periyasamy ${ }^{2}$ \\ ${ }^{I}$ (Research Scholar, Sree Saraswathi Thyagaraja College,Thippampatti,India) \\ ${ }^{2}$ (Assistant Professor in MCA,Department of Master of Computer Applications Sree Saraswathi Thyagaraja \\ College, Thippampatti,India.)
}

\begin{abstract}
Disruption tolerant networks (DTNs) are characterized by low node density, unpredictable node mobility, and lack of global network information. Most of current research efforts in DTNs focus on data forwarding, but only limited work have been done on providing efficient data access to vehicle users. The proposed Fuzzy Probabilistic Routing Protocol using History of Encounters and Transitivity (FPRoPHET) protocol uses an algorithm that attempts to exploit the non-randomness of real-world encounters by maintaining a set of probabilities for successful delivery to known destinations in the VDTN (delivery predictabilities) and replicating messages during opportunistic encounters only if the Mule that does not have the message appears to have a better chance of delivering it. Propose a novel approach to support fairness aware cooperative caching scheme in VSNs (Vehicle Social Networks). Through capturing close friend set of each node, we cache data prior at nodes which are overlapped by most nodes' close friend sets. Proposed system develop an optimization procedure to restrict the scope of caching nodes to satisfy the condition that there is at least one common node between caching node set and each node's close friend set. By this mean, we minimize the number of caching nodes while ensuring the fairness of each node at the same time. Extensive trace-driven simulations show that our approach significantly improves data access performance compared to existing schemes.
\end{abstract}

Keywords: DTN; Routing Protocol; Fuzzy Logic; VANET.

\section{Introduction}

DTNs are a special class of networks that allow communication between regions with strong connectivity constraints, propagation delays and high error rates. In these regions, there is no way to use reliable communication and routing protocols or common standards for wireless networks as has been shown by Sadagopan et al. [1]. To overcome these constraints, the RFC4838 [2] propose that the DTN architecture can store persistent messages in a new network layer named the bundle layer until the node connectivity could be restored. This layer can provide communication between heterogeneous networks operating at different transmission media. Despite this clear advantage of MANETs, they are not used in practice, in part because there are not the tools and methodologies necessary for network planners and engineers to reason about the performance of real-world MANET deployments. Analysis of such networks is challenging due to the time variability of the network as induced by the mobility and link volatility.

Many researchers have proposed new routing protocols such as Epidemic "[5]", Prophet "[6]", Sprayand-Wait "[7]", Spray-and-wait "[8]", Max Prop "[9]", ORWAR "[10]”, ERS “[11]", APRP “[12]", and PFBR "[13]" to handle this specific problem for DTN.

Delay-tolerant networks (DTN) try to extend the reach of networks. It promises to enable communication between challenged networks, which includes space networks, mobile ad-hoc networks, and low cost networks. The core idea is that these networks can be connected if protocols are designed to accommodate disconnection. Vehicular Delay-Tolerant Networks have the potential to interconnect Vehicles in regions that current networking technology cannot reach. The main core is that an end-to-end connection may never be reached. To make end-to-end communication possible, relay nodes take the advantage of mobility for the data being transferred and forward it as the opportunity arises.

The DTN architecture implements a store-and forward paradigm by overlaying a protocol layer, called bundle layer that it is meant to provide internetworking on heterogeneous networks operating on different transmission media . At the edge of each remote area network, a border system has an application layer gateway to terminate applications and produce data bundles. The DTN architecture concept was also extended to transit networks, called Vehicular DTN (VDTN). In these networks vehicles (e.g., cars, buses, and boats) are exploited to offer a message relaying service by moving around the network and collecting messages from source nodes. A number of projects have been based on this general concept. For example, the Message Ferry project to develop a data delivery system in disconnected areas [3,4]. Another example is the DakNet project proposed to provide low-cost connectivity to the Internet to rural villages in India. Vehicular Networks have also been used 
for traffic condition notifications, accident warnings, automatic tolling, free parking spots information, advertisements, and for example to gather data collected by vehicles like road pavement defects.

Traditional routing protocols are not suitable for this scenario, because in those routing protocols, endto-end connection between the source and the destination node is basic assumption. Devices in opportunistic network are enabled to interconnect by operating message in a store-carry-forward style and, each node can act as host, intermediate node, thus, it can store, carry and forward the message between for other nodes. The big challenge in opportunistic networks is how to route messages from their source to their destination, with the absence of end-to-end path. When there is no path existing between the source and the destination, nodes need to communicate with each other via opportunistic contacts through store-carry-forward operation.

In this paper simulation of concrete protocols for these vehicle delay tolerant networks (FProPHET) allows us to understand the performance of these protocols in a given vehicle mobility scenario. Although simulation of these protocols is novel, applying the results obtained from these simulations in the context of achievable performance of these networks is new and provides an understanding of the performance seen in the simulation. For example, the high performance achieved by an FProPHET protocol in a given network scenario could be due to the properties of the protocol or due to the fundamental properties of the network itself (e.g., the network is rarely connected). In these networks vehicles use a message relaying service by their mobility in the network and collect messages from source nodes. In this paper, we will review the routing protocol in delay tolerant networks and compare the routing protocols that have been proposed specially for Vehicular Delay Tolerant Networks.

The rest of the paper is organized as follows. Section 2, gives an overview of related work. Section 3 presents proposed approach. In Section 4, deal with some topologies to validate proposed approach. Conclusion is presented in Section 5.

\section{Related Works}

A VANET environment has characteristics that hinder the existence of an end-to-end path between source and destination; therefore, DTN routing protocols have been designed for The VDTN- FProPHET uses the metric FProPHET to assist in routing decisions to allow a particular network node to decide when it is best to keep, forward or copy a packet, taking into account improvements in the delivery rates and decrease in the message delays. The VDTN also uses a scheme of disclosure and maintenance of location messages based on the concept of Adaptive Coverage Detection (ACD), which takes into account the transmission range, to reduce the number of update messages with their location details given by the nodes.

$\mathrm{Yu}$ and Ko [14] proposed the VANET/DTN protocol called Fastest-Ferry Routing in DTN-enabled Vehicular Ad Hoc Networks (FFRDV). It works specifically in motor-highway scenarios. It divides the highway into blocks and within them it decides to which vehicle as a relay node it will forward the packet, based on the vehicle speed. The FProPHET that we incorporated into the DTN routing protocols in our work reported in this paper also uses the speed factor to assist in the routing decisions, but we go one step further taking into account the angle between the vehicle and the distance to the target node.

Zhao and Cao in [15] proposed the protocol Vehicle-Assisted Data Delivery in Vehicular Ad Hoc Networks (VADD) that uses a digital map to obtain the maximum speed, the vehicle density and intersection places. Based on this information, it uses a metric called expected delay for delivery to make routing decisions when one arrives at an intersection/junction. When it is not at an intersection it typically works like Greedy Perimeter Stateless Routing (GPSR) [16]. The VADD does not perform packet replication, but forwarding, and furthermore the target nodes are fixed in the proposed application. In our proposed scheme in the FProPHET protocols reported in this paper, we go one step further to ensure knowing each target's geo location of the vehicle nodes, which are e evaluated on their mobility.

Another VANET/DTN protocol that uses metrics for its routing decisions is the Geographical Opportunistic Routing for Vehicular Networks (GeOpps) [17], which is obtained with the aid of a navigation system that makes a node to know the routes of its neighbors in it vicinity range. Since the navigation system indicates which way the neighbors will take (based on their source-to-destination route selection), suggest that GeOpps may achieve more optimum routing than in other protocols with FProPHET. However, this scheme may expose user security and privacy that could be used by criminals and other agencies for the wrong reasons. Protocols with FProPHET have the advantage of requiring less information from the VANET environment. Another proposal GeoSpray [18], which is a combination of GeOpps with Spray-And-Wait extends.

\subsection{Epidemic Routing}

Epidemic Routing is classified as replication-based routing. In Epidemic Routing, each node distributes replicated messages with no restrictions. In simple word, every node forwards their stored messages to each meeting node. Epidemic routing each node send a duplicate message with no limit so it is generate more traffic on network Epidemic Routing is the most avoidable in all DTN routings. 


\subsection{Spray and wait}

Spray and Wait protocol work on controlled copy/Replication Schemes. There are different scheme for its protocol like Spray and wait (Snw) its advantage fewer transmission than epidemic, low contention under high traffic, scalable, Requires little knowledge about network and Disadvantage is only source node is allowed to spray copies. Thus, it incurs considerable Delay \& needs to investigate the performance in realistic situation. Binary Spray and Wait (BSW) its Advantage: Fewer transmissions than epidemic, low contention under high traffic, scalable, Requires little knowledge about network and It does blind fold forwarding (random) of message copies. It needs to investigate the performance in realistic situation.Spray and Focus has advantage are Improves the performance by twenty times than spray \& wait, Disadvantage Finding optimal distribution strategy, Spray and Wait with average delivery probability, Fuzzy Spray and Wait its has own.

\subsection{MaxProp}

MaxProp is forwarding based routing protocol. In MaxProp routing each node initially set a probability of meeting to all the other nodes in network and also exchanges these values to its neighbour nodes. The probability value is used to calculate a destination path cost. Each node forwards messages through the lowest cost path. MaxProp also uses an ordered queue which is divided into two parts according to an adaptive threshold. MaxProp assigns a higher priority to new messages and forward it first with low hop count and drops a message with the highest cost path when buffer is full. MaxProp has poor performance when nodes have small buffer sizes because of the adaptive threshold calculation. Max Prop performance is better with large buffer size.

\section{Proposed Approach}

Besides the VDTN uses this mobility information, it also uses fuzzy logic to identify mobility patterns and then decides when it is appropriate to: copy, forward or stay with the message. Propose PROPHET (Probabilistic Routing Protocol using History of Encounters and Transitivity), a single copy history-based routing algorithm for DTNs. Each node in PROPHET estimates a delivery predictability vector containing an entry for each other node. A probabilistic metric called delivery predictability estimates the probability that node A will be able to deliver a message to node B. The delivery predictability vectors are maintained at each node A for every possible destination B. Predictability vectors will be used to decide on packet forwarding. When two nodes contact each other, node if the delivery predictability for the destination of the message is higher at the other node, a message is forwarded to the other. In addition to the predictability vector, a summary vector of stored packets will be also exchanged upon contact.

The information in the summary vector is used to decide on which messages to request from the other node. The entry update process happens whenever each contact and works as follows. Nodes that are often within mutual ranges have high delivery predictability for each other, and as they increase their corresponding delivery predictability entries. Nodes that rarely connect are less likely to be good forwarders of messages to each other; therefore they will reduce their corresponding delivery predictability entries.

\subsection{Delay-Tolerant Routing in VANETs}

Although most of the existing work on vehicle networks is limited to 1-hop or short range multi-hop communication, vehicular delay-tolerant networks are useful to other scenarios. For example, without Internet connection, a moving vehicle may want to query a data center ten miles away through a VANET. The widely deployed wireless LANs or info stations can also be considered. Vehicle delay-tolerant networks have many applications, such as delivering advertisements and announcements regarding sale information or remaining stocks at a department store. Information such as the available parking spaces in a parking lot, the meeting schedule at a conference room, and the estimated bus arrival time at a bus stop can also be delivered by vehicle delay-tolerant networks.

For the limited transmission range, only clients around the access point can directly receive the data. However, this data may be beneficial to people in moving vehicles far away, as people driving may want to query several department stores to decide where to go. A driver may query the traffic cameras or parking lot information to make a better travel plan. A passenger on a bus may query several bus stops to choose the best stop for bus transfer. All these queries may be issued miles away from the broadcast site. With a vehicular delay-tolerant network, the requester can send the query to the broadcast site and get a reply from it. In these applications, the users can tolerate up to a minute of delay as long as the reply eventually returns. In this section we will review the delay tolerant protocols specially proposed for Vehicular Networks.

\subsubsection{Vehicle Assisted Data Delivery}

That is, nodes can decide to keep the message until a more promising neighbor appears on their coverage range, but trying always to forward them as soon as possible. Additionally, decisions about which streets must be followed by the packet are made using vehicle and road information such as current speed, 
distance to the next junction, and maximum speed allowed. These routing decisions are dynamically taken at junctions because the authors state that pre-computed optimal paths used by other protocols might rapidly lose their optimality due to the unpredictable nature of VANETs. In VADD the main goal is to select the path with the smallest packet delivery delay. The behavior of the protocol depends on the location of the node holding the message. Two cases are considered: when nodes routing the message are located in the middle of a road and when they are located in a junction. The first case (also called routing in straight way) presents less alternatives: forwarding the packet toward the next junction or to the previous one. However, the second case (also called routing in intersections) is much more complicated because at junctions, the routing decision must consider the different roads, so that the number of options is higher.

\subsection{Fuzzy spray based Routing Protocol}

Although the random way-point mobility model is popular to use in evaluations of mobile ad hoc protocols, real users are not likely to move around randomly, but rather move in a predictable fashion based on repeating behavioral patterns such that if a node has visited a location several times before, it is likely that it will visit that location again. We would like to make use of these observations and this information to improve routing performance by doing probabilistic routing and thus, we propose FPROPHET, a Fuzzy Probabilistic Route cache Protocol using History of Encounters and Transitivity. To accomplish this, we establish a probabilistic metric called delivery predictability, $\mathrm{P}(\mathrm{a} ; \mathrm{b}) 2[0 ; 1]$, at every node a for each known destination $\mathrm{b}$. This indicates how likely it is that this node will be able to deliver a message to that destination. The operation of FPROPHET is similar to that of Epidemic Routing. When two nodes meet, they exchange summary vectors which in this case also contain the delivery predictability information stored at the nodes. This information is used to update the internal delivery predictability vector as described below, and then the information in the summary vector is used to decide which messages to request from the other node based on the forwarding strategy used.

\subsubsection{Probability of Delivery}

In FPRoPHET (Fuzzy Probabilistic Routing Protocol using History of Encounters and Transitivity), before sending a message, each node estimates a probabilistic metric called Delivery Predictability for each known destination. It indicates the probability of successful delivery of a message to the destination from the source node. The calculation of the Delivery Predictability is based on the history of encounters between nodes or history of visits to certain locations. This metric is calculated by each node A of the DTN network and for each known destination $\mathrm{B}$ and will be used to decide which messages to be exchanged whenever two nodes meet.

The calculation of the probability of delivery is done in three steps: When a node A meets another node B: A updates the probability of delivery in accordance with the following equation:

$$
\mathbf{P}_{(\mathbf{a}, \mathbf{b})}=\mathbf{P}_{(\mathbf{a}, \mathrm{b}) \text { old }}+\left(\mathbf{1}-\mathbf{P}_{(\mathbf{a}, \mathrm{b}) \text { old }}\right) \times \mathbf{P}_{\text {init }}
$$

If a pair of nodes does not encounter each other in a while, they are less likely to be good forwarders of messages to each other, thus the delivery predictability values must age, being reduced in the process. The aging equation is shown in Eq. 2, where $\gamma \in[0,1]$ is the aging constant, and $\mathrm{k}$ is the number of time units that have elapsed since the last time the metric was aged. The time unit used can differ, and should be defined based on the application and the expected delays in the targeted network.

$$
\mathbf{P}_{(\mathbf{a}, \mathbf{b})}=\mathbf{P}_{(\mathbf{a}, \mathbf{b}) \mathbf{o l d}} \times \gamma^{\mathrm{k}}
$$

The delivery predictability also has a transitive property, that is based on the observation that if node $\mathrm{A}$ frequently encounters node $\mathrm{B}$, and node $\mathrm{B}$ frequently encounters node $\mathrm{C}$, then node $\mathrm{C}$ probably is a good node to forward messages destined for node A to. Eq. 3 shows how this transitivity affects the delivery predictability, where $\beta \in[0,1]$ is a scaling constant that decides how large impact the transitivity should have on the delivery predictability.

$$
\mathbf{P}_{(\mathbf{a}, \mathbf{c})}=\mathbf{P}_{(\mathbf{a}, \mathrm{c}) \text { old }}+\left(1-\mathbf{P}_{(\mathbf{a}, \mathrm{c}) \text { old }}\right) \times \mathbf{P}_{(\mathrm{a}, \mathrm{b})} \times \mathbf{P}_{(\mathrm{b}, \mathrm{c})} \times \boldsymbol{\beta}
$$

Where is calculated in the following way:

$$
\mathbf{P}_{\mathrm{enc}}=\mathbf{P}_{\mathrm{enc}-\max }\left(\operatorname{Intvl}_{\mathrm{B}} / \mathbf{I}_{\mathrm{typ}}\right.
$$

For intvl $_{\mathbf{B}} \in\left[\mathbf{0}, \mathbf{I}_{\text {typ }}\right]$

$$
=\mathbf{P}_{\text {enc- }} \text { max }
$$

Where is used as the upper limit of scaling factor that increases the delivery predictability for a destination when the destination node is encountered, is a parameter that is set to the expected typical time interval between connections in the application scenario relevant to the network deployment and is the time since the last en-counter with node B. Each node maintains in its cache the information of all the nodes it has contacted recently i.e. the frequency of encounters with each node and thus a probability of delivery value will be associated. 


\subsection{Fuzzy Prophet Routing Implementation}

Our approach to routing is based on the frequency of contacts between network nodes. Therefore, each network node calculates the probability of delivery (As it is mentioned above, from equation 1 to equation 5). The measured probability of delivery and energy value is used as input to the fuzzy system in order to compute the delivery predictability value which determines the routing path for packets. In this regards the defined inputs and output sets have been defined for FBRP protocol.

\subsubsection{Probability of Delivery (PD)}

The most important problem of fuzzy logic is to define the appropriate ranges for each fuzzy input. On the bases of recommended parameter values, we can define the range for probability of delivery. The membership function of PD is divided into 3 sections, low, medium and high, with linear symmetric shape. Figure 3.2 illustrates the degree of membership function of PD.

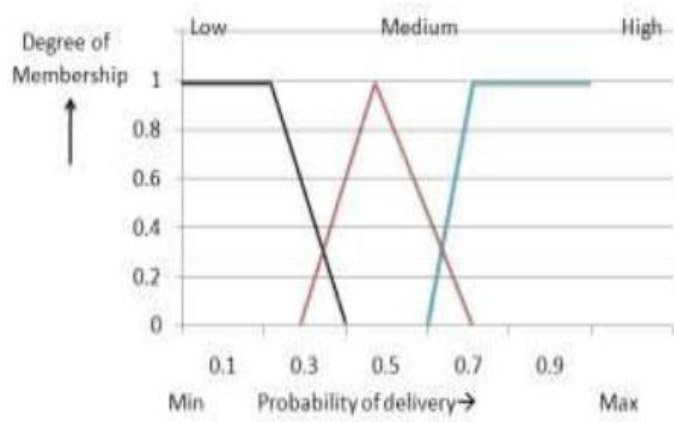

Fig. 3.1 Memberships function of probability of delivery.

\subsubsection{Energy Value (EV)}

We defined that every node is in high level which means it has full capacity $(100 \%)$. The node will not be a good router to forward the packets if the energy of it falls below 50\%. For energy capacity between $50 \%$ to $100 \%$ of total apacity, we define high fuzzy set, for $20 \%$ to $80 \%$ we define medium fuzzy set and for $0 \%$ to $50 \%$ we define low fuzzy set.

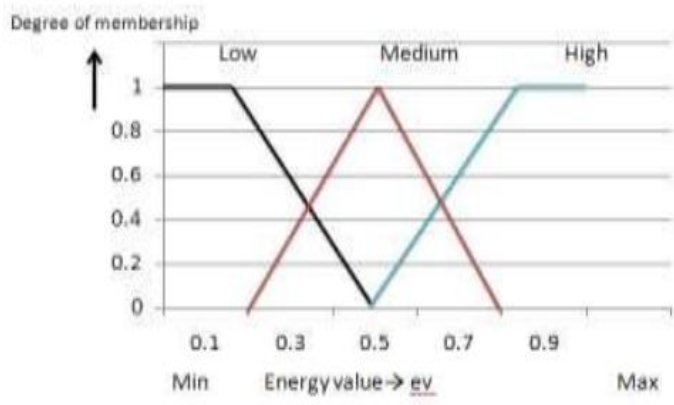

Fig. 3.2 Memberships function of energy value.

\subsubsection{Delivery Predictability (DP) Evaluation}

The membership functions of delivery predictability. It takes a different values based on 9 rules that dependent upon varied input metric values i.e. PD and EV. A fuzzy system decides for each two input values which values appear in output.

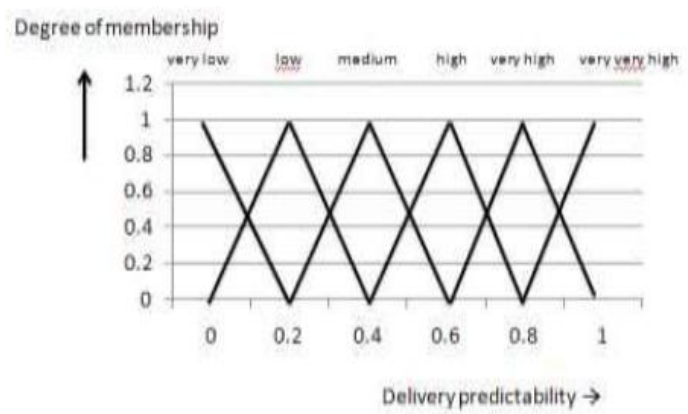

Fig. 3.3 Memberships function of delivery predictability. 
The fuzzy system with product inference engine, singleton fuzzifier and center average defuzzifier i s of the following form:

$$
\mathbf{f}(\mathbf{x})=\frac{\sum_{\mathbf{i}=\mathbf{1}}^{\mathbf{6}} \mathbf{y}_{\mathbf{0}}^{\mathrm{i}}\left(\prod_{\mathbf{k}=\mathbf{1}}^{2} \boldsymbol{\mu}_{\mathrm{A}_{\mathrm{ik}}}\left\{\mathbf{x}_{\mathbf{k}}\right\}\right)}{\sum_{\mathbf{i}=\mathbf{1}}^{\mathbf{C}} \prod_{\mathbf{k}=\mathbf{1}}^{2} \boldsymbol{\mu}_{\mathrm{A}_{\mathrm{ik}}}\left\{\mathbf{x}_{\mathbf{k}}\right\}}
$$

where $y_{0}^{i}$ is the center average of output fuzzy set, $x_{i}$ represents crisp value of $i^{\text {th }}$ input (probability of delivery or energy value and $\mu_{\mathrm{A}_{\mathrm{ik}}}\left(\mathrm{x}_{\mathrm{k}}\right)$ represents fuzzy membership function for $\mathrm{i}^{\text {th }}$ input.

The fuzzy rules are as follows:

1. If PD is low and $\mathrm{EV}$ is low then DP is very low

2. If $\mathrm{PD}$ is medium and $\mathrm{EV}$ is low then $\mathrm{DP}$ is very low

3. If $\mathrm{PD}$ is high and $\mathrm{EV}$ is low then DP is very low

4. If $\mathrm{PD}$ is low and $\mathrm{EV}$ is medium then $\mathrm{DP}$ is low

5. If $\mathrm{PD}$ is medium and $\mathrm{EV}$ is medium then $\mathrm{DP}$ is medium

6. If $\mathrm{PD}$ is high and $\mathrm{EV}$ is medium then $\mathrm{DP}$ is high

7. If $\mathrm{PD}$ is low and $\mathrm{EV}$ is high then $\mathrm{DP}$ is very low

8. If $\mathrm{PD}$ is medium and $\mathrm{EV}$ is high then $\mathrm{DP}$ is very high

9. If $\mathrm{PD}$ is high and EV is high then DP is very very high

\section{Experimental Results}

To validate behavior and evaluate the performance of FBRP, we use ONE (Opportunistic Network Environment) simulator to implement it and other DTN routing protocols, including Epidemic and FPRoPHET. In our simulation, we u s e d the real world connectivity and traffic traces, collected during a N4C deployment in 2010. The traces come from one week when the mobility conditions resembled the ones in the N4C summer test of 2009. During that period ( July 27-August 3, 2010) only one data mule carrier was used and the network had high usage. The total number of DTN nodes was 18 and the number of bundles sent was 1407. The lifetime of these bundles was set to three days. Only one helicopter flight with data mules was scheduled per day and some of the bundles that were sent in the last days had to be dropped at the end of simulations. FPRoPHET could deliver the same number of bundles as Epidemic Routing with a lower overhead. Moreover, FBRP (The proposed routing) could deliver a higher number of bundles with minimum overhead rather than PRoPHET and Epidemic Routing.

In the second evaluation we used the Working Day Model (WDM) with the default settings for WDM in the ONE simulator. The simulation time was set to one week and the expiry time for the bundles was set to 1430 minutes. The number of nodes was 500, and a total of 17000 bundles were sent. In this set up, the buffer space was limited to $100 \mathrm{MB}$ per node and the available bandwidth was $100 \mathrm{kbit} / \mathrm{s}$. As seen in Figure 5, despite limited resources (buffer and band- width) FBRP (The proposed routing) performed better performance than FPRoPHET and Epidemic Routing in this scenario Delivery rate and overhead ratio. The fact that the proposed routing outperformed FPRoPHET and Epidemic Routing shows that the protocol makes wise decisions on what bundles to forward and how to use the limited resources.

\section{Performance Comparisons}

Our simulations produced three sets of results using the traces of the buses for determining when transfer opportunities occur and for how much bandwidth is transferred at each. First, we analyzed three specific scenarios:

1) Delivery rate and latency when offered load varies from 2 to 18 packets / hour on each bus (with fixed buffer size and packet size). These values represent the means of exponentially distributed packet loads.

2) Delivery rate and latency when buffer size varies from $500 \mathrm{~KB}$ to $5 \mathrm{MB}$ on each bus (with fixed offered load and packet size).

3) Delivery rate and latency when packet size increases from 10KB to 100KB (with fixed buffer size and offered load).

Then using synthetic traces, we analyzed how the protocols performed when the number of buses in the network varied, and how the protocols performed with different radio ranges.

Table 5.1 PACKet Delivery RATIO

\begin{tabular}{|l|l|l|l|l|l|}
\hline \multirow{2}{*}{ Protocols } & \multicolumn{6}{|l|}{ Buffer size } \\
\cline { 2 - 6 } & $\mathbf{2}$ & $\mathbf{4}$ & $\mathbf{6}$ & $\mathbf{8}$ & $\mathbf{1 0}$ \\
\hline Epidemic & 0.34 & 0.43 & 0.5 & 0.6 & 0.73 \\
\hline PRoPHET & 0.43 & 0.45 & 0.58 & 0.73 & 0.79 \\
\hline Spray And Wait & 0.51 & 0.57 & 0.61 & 0.85 & 0.84 \\
\hline Proposed FPRoPHET & $\mathbf{0 . 6 4}$ & $\mathbf{0 . 7}$ & $\mathbf{0 . 8 4}$ & $\mathbf{0 . 9 5}$ & $\mathbf{1 . 0}$ \\
\hline
\end{tabular}




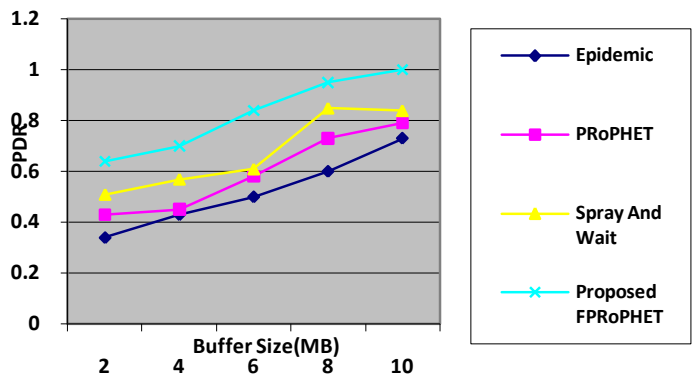

Fig. 5.1 Comparison of different protocol vs Packet delivery ratio

Fig 5.1 shows packet delivery ratio against Buffer size. It shows that the Proposed FPRoPHET protocol has a better throughput in the different size of buffer.

Table 5.2 AVERAGE LATENCY

\begin{tabular}{|l|l|l|l|l|l|}
\hline \multirow{2}{*}{ Protocols } & \multicolumn{5}{|l|}{ Buffer size } \\
\cline { 2 - 6 } & $\mathbf{2}$ & $\mathbf{4}$ & $\mathbf{6}$ & $\mathbf{8}$ & $\mathbf{1 0}$ \\
\hline Epidemic & 4673 & 4765 & 5873 & 6894 & 8435 \\
\hline PRoPHET & 3745 & 3646 & 4638 & 5873 & 7874 \\
\hline Spray And Wait & 2547 & 2896 & 3689 & 4876 & 6474 \\
\hline Proposed FPRoPHET & $\mathbf{1 4 5 7}$ & $\mathbf{2 6 4 3}$ & $\mathbf{3 4 5 7}$ & $\mathbf{4 3 2 8}$ & $\mathbf{5 7 5 7}$ \\
\hline
\end{tabular}

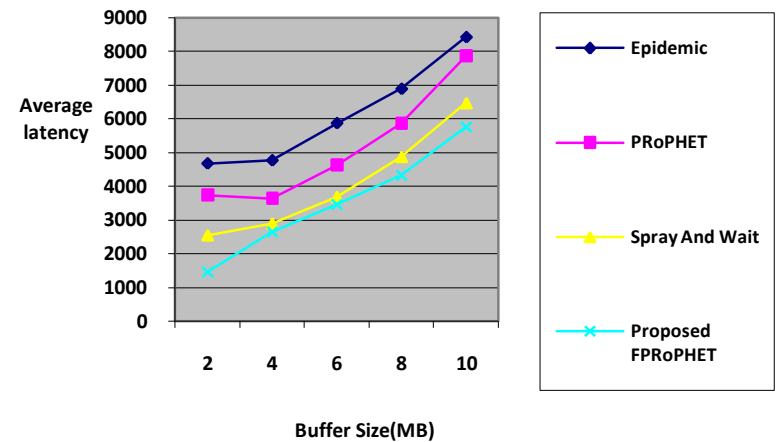

Fig. 5.2 Average latency as function of buffer size.

Fig. 6 shows the average delay of a message as the buffer size varies. Similar to the delivery ratio, the result shows that the performance of FPRoPHET is better than those of PRoPHET, and Epidemic.

Table 5.3 OvERHEAD RATIO

\begin{tabular}{|c|c|c|c|c|c|}
\hline \multirow[t]{2}{*}{ Protocols } & \multicolumn{5}{|c|}{ Buffer size } \\
\hline & 2 & 4 & 6 & 8 & 10 \\
\hline Epidemic & 77 & 66 & 55 & 52 & 48 \\
\hline PRoPHET & 68 & 61 & 48 & 45 & 41 \\
\hline Spray And Wait & 59 & 52 & 43 & 39 & 36 \\
\hline Proposed FPRoPHET & 51 & 46 & 38 & 35 & 31 \\
\hline
\end{tabular}

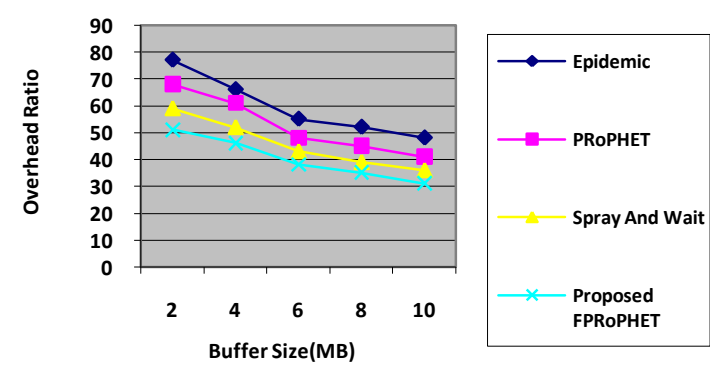

Fig. 5.3 overhead ratios as function of buffer size 
Fig.5.3 Fuzzy PRoPHET the simulation results indicate, the copies of each message are much less than PRoPHET and Epidemic Routing protocols.

\section{Conclusions And Future Work}

Proposed fuzzy-based routing protocol, called FPROPHET. It uses only two parameters namely, probability of delivery and energy value as input to fuzzy system in order to compute the delivery predictability value which determine the routing path for packets. During transmission it is needed only to pass delivery probability along with the actual message to the peer. The fuzzy membership functions can be adaptively constructed based on known network parameters. The fuzzy decision mechanism is very simple compared to complex prediction mechanisms used in many other DTN protocols. In spite of that, we have shown that FPROPHET is the best performing protocol, in terms of delivery rate and overhead ratio. Thus, it is fair to say that FPROPHET succeeds in its goal of providing communication opportunities to entities in a intermittently connected network with a lower communication overhead, and better performance than existing protocols. The future work to reduce the required buffer space, and to further improve performance, it would be interesting to evaluate the impact of allowing nodes to request an ACK to their message. This would allow messages that already have been delivered to be purged from the network, leaving more resources for the other messages, most likely increasing the probability of those messages being delivered.

\section{References}

[1] N. Sadagopan, f. Bai, b. Krishnamachari, and a. Helmy, "paths: analysis Of path duration statistics and their impact on reactive manet routing Protocols," in proceedings of the 4th acm international symposium on Mobile ad hoc networking \& computing. Acm, 2003, pp. 245-256.

[2] V.Cerf et al. "rfc 4838, delay-tolerant networking architecture," irtf Dtn research group, 2007.

[3] W.Zhao, m. Ammar, and e. Zegura, "a message ferrying Approach for data delivery in sparse mobile ad hoc networks," In the fifth acm international symposium on mobile ad hoc Networking and computing (mobihoc 2004), roppongi hills, Tokyo, japan, may 24 26, 2004, pp. 187-198.

[4] H.Ammar, "message ferrying: proactive routing in highly-Partitioned wireless ad hoc networks," in the ninth ieee Workshop on future trends of distributed computing systems, San juan, puerto rico, may 28-30, 2003, pp. 308-314.

[5] A.Vahdat and d. Becker, "epidemic routing for partially connected ad hoc networks", tech. Rep. Cs-2000-06, cs dept., duke university, april 2000.

[6] A.Lindgren et al, "probabilistic routing in intermittently connected networks", mobile comp. And comm. Rev, vol. 7, no. 3, pp. 1920 , july 2003

[7] T.Spyropoulos, k. Psounis, and c. S. Raghavendra, "spray and wait: efficient routing in intermittently connected mobile networks", in proceedings of acmsigcomm workshop on delay tolerant networking (wdtn'5), pp 252-259, 2005.

[8] J.Burgess, b. Gallagher, d. Jensen and b. N. Levine, "maxprop: routing for vehicle-based disruption-tolerant networks," proceedings of 25th ieee international conference on computer communications, barcelona, 23-29 april 2006, pp. 1-11. Doi:10.1109/infocom.2006. 228

[9] J.Lebrun, c.-n. Chuah, d. Ghosal, and m. Zhang, "knowledgebased opportunistic forwarding in vehicular wireless ad hoc networks," in ieee vehicular technology conference(vtc), pp. 2289-2293, may 2005.

[10] J.Leguay, t. Friedman, v. Conan, "dtn routing in a mobility pattern space", presented at acm sigcomm workshop on delay tolerant networking, 2005

[11] Hui, p. And crowcroft, j. (2007) "bubble rap: forwarding in small world dtns in every decreasing circles", technical report, technical report ucam-cl-tr684. Cambridge, uk: university of cambridge.

[12] Boldrini, c., conti, m., jacopini, i., \& passarella, a.(2007, june). "hibop: a history based routing protocol for opportunistic networks". Paper presented in the proceedings of the wowmom 2007, helsinki.

[13] Hemal shah and yogeshwar. P. Kosta , "routing enhancement specific to mobile environment using dtn", international journal of computer theory and engineering, vol. 3, no. 4, august 2011

[14] D.Yu and y. Ko, "ffrdv: fastest-ferry routing in dtn-enabled vehicular Ad hoc networks," in advanced communication technology, 2009.Icact 2009. 11th international conference on, vol. 2. Ieee, 2009, Pp. 1410-1414.

[15] J.Zhao and g. Cao, "vadd: vehicle-assisted data delivery in vehicular," Vehicular technology, ieee transactions on, vol. 57, no. 3, pp. 1910-1922, 2008

[16] B.Karp and h.-t. Kung, "gpsr: greedy perimeter stateless routing For wireless networks," in proceedings of the 6th annual international Conference on mobile computing and networking. Acm, 2000, pp. 243-254.

[17] I.Leontiadis and c. Mascolo, "geopps: geographical opportunistic Routing for vehicular networks," in world of wireless, mobile and multimedia Networks, 2007. Wowmom 2007. Ieee international symposium On a. Ieee, 2007, pp. 1-6.

[18] V. N. Soares, j. J. Rodrigues, and f. Farahmand, "geospray: a geographic Routing protocol for vehicular delay-tolerant networks," information Fusion, 2011. 